\title{
The Alternative Operad Is Not Koszul
}

\author{
Askar Dzhumadil'daev and Pasha Zusmanovich
}

\section{CONTENTS}

1. The Alternative Operad and Its Koszul Dual

2. Dimension Sequence

3. Non-Koszulity

4. Positive Characteristic

5. Questions

Acknowledgments

References
2000 AMS Subject Classification: Primary 17D05; Secondary 05A05, 16N40, 16S37, 17-04, 17DI5, 18D50, 65F99

Keywords: alternative operad, right-alternative operad, Koszulity, Poincaré series
In the online compendium [Loday 07], it is asked whether the alternative operad is Koszul. The purpose of this note is to demonstrate that the answer to this question is negative. In doing so, we have been aided by the programs Albert and PARI/GP.

\section{THE ALTERNATIVE OPERAD AND ITS KOSZUL DUAL}

Recall that an algebra is called right-alternative if it satisfies the identity

$$
(x y) y=x(y y)
$$

and left-alternative if it satisfies the identity

$$
(x x) y=x(x y) \text {. }
$$

An algebra that is both right-alternative and leftalternative is called alternative.

Linearizing identities (1-1) and (1-2), we get

$$
(x y) z+(x z) y-x(y z)-x(z y)=0
$$

and

$$
(x y) z+(y x) z-x(y z)-y(x z)=0,
$$

respectively. If the characteristic of the ground field is different from 2 , these identities are equivalent to the initial ones, and they define binary quadratic operads $\mathcal{R} \mathcal{A} l t$, $\mathcal{L} \mathcal{A} l t$, and $\mathcal{A}$ lt (called right-alternative, left-alternative and alternative operads). In characteristic 2 things go berserk: identities (1-1) and (1-2) are not equivalent to the corresponding linearized identities, so it is impossible to encode them with operads in a straightforward manner. We will exclude this case from our considerations.

Right- and left-alternative algebras are opposite to each other in the sense that if $A$ is a right-alternative algebra, then the algebra defined on the same vector space $A$ with multiplication $x \circ y=y x$ is a left-alternative algebra, and vice versa. Hence all the statements below for left-alternative algebras automatically follow from the corresponding statements for right-alternative ones, and 
in the proofs we will consider the right-alternative case only. Most of these statements are trivial and/or have been considered previously in the literature, but they provide a good warmup before the more difficult alternative case.

Every associative algebra is alternative. An example of a nonassociative alternative algebra is the octonion algebra, appearing prominently in mathematics and physics (see, for example, the excellent survey [Baez 02]). Note also that free alternative algebras are much more difficult objects than, for example, their associative or Lie counterparts, and are still not understood sufficiently well.

For the general operadic business, including the important notions of Koszulity and Koszul duality, we refer to the book [Markl et al. 02] and the foundational paper [Ginzburg and Kapranov 94]. However, to understand this note it is enough to adopt an intuitive and primitive view on operads as polylinear parts of the corresponding free algebras, and to accept the Ginzburg-Kapranov criterion for Koszulity, as described below, for granted.

Proposition 1.1. Each of the operads Koszul dual to the right-alternative, left-alternative, and alternative operad is defined by two identities: associativity and the identity

$$
x y z+x z y=0
$$

in the right-alternative case,

$$
x y z+y x z=0
$$

in the left-alternative case, and

$$
x y z+y x z+z x y+x z y+y z x+z y x=0
$$

in the alternative case.

In the alternative case, this is stated in [Loday 07] without proof, so we will provide a simple (and pretty much standard for such situations) proof for completeness. Following [Loday 07], we will call algebras over the corresponding Koszul dual operads dual rightalternative, dual left-alternative, and dual alternative, respectively.

Proof: Let $R$ be the space of quadratic relations of the alternative operad, i.e., the space generated by the lefthand sides of identities (RA) and (LA), and let $R^{\perp}$ be the space of quadratic relations of the dual alternative operad.

Identities (RA) and (LA) imply that we may take the images of the seven monomials $(x y) z,(y x) z,(x z) y,(z x) y$, $(y z) x,(z y) x, z(x y)$ as the basis of $\mathcal{A l t}(3)$, with the re- maining monomials expressed through them as follows:

$$
\begin{aligned}
& z(y x)=(z x) y+(z y) x-z(x y), \\
& y(z x)=-(z x) y+(y z) x+z(x y), \\
& y(x z)=(y x) z+(z x) y-z(x y), \\
& x(y z)=(x y) z-(z x) y+z(x y), \\
& x(z y)=(x z) y+(z x) y-z(x y) .
\end{aligned}
$$

In particular,

$$
\operatorname{dim} \mathcal{A} l t(3)=\operatorname{dim} R^{\perp}=7
$$

and

$$
\operatorname{dim} \mathcal{A} l t(3)=\operatorname{dim} R=3 ! C_{2}-7=5
$$

(here and below,

$$
C_{n}=\frac{(2 n) !}{n !(n+1) !}
$$

denotes the $n$th Catalan number).

To obtain identities defining the dual alternative operad, it is convenient to use the fact that if $L$ is an alternative algebra and $A$ is a dual alternative algebra, then their tensor product $L \otimes A$ equipped with the bracket

$$
[x \otimes a, y \otimes b]=x y \otimes a b-y x \otimes b a
$$

for $x, y \in L, a, b \in A$, is a Lie algebra. Writing the Jacobi identity for triple $x \otimes a, y \otimes b, z \otimes c$ for $x, y, z \in L$, $a, b, c \in A$, substituting in it all equalities (1-3), and collecting similar terms, we get

$$
\begin{aligned}
& (x y) z \otimes((a b) c-a(b c))+(y x) z \otimes(b(a c)-(b a) c) \\
& +(x z) y \otimes(a(c b)-(a c) b) \\
& +(z x) y \otimes(a(b c)+a(c b)+b(a c)+b(c a)+(c a) b+c(b a)) \\
& +(y z) x \otimes((b c) a-b(c a))+(z y) x \otimes(c(b a)-(c b) a) \\
& -z(x y) \otimes(a(b c)+a(c b)+b(a c)+b(c a)+c(a b)+c(b a)) \\
& \quad=0
\end{aligned}
$$

and the claimed identities follow.

Now it is straightforward to check that the relations so obtained are orthogonal to the alternative relations $R$ under the standard pairing (as defined in [Ginzburg and Kapranov 94, Section 2.1.11]), so they really lie in $R^{\perp}$. Under the action of the symmetric group $S_{3}$, the associativity gives six different relations, and the left-hand side of $\left(\mathrm{A}^{!}\right)$is $S_{3}$-invariant, so we get seven relations in total. This shows that all relations have been taken into account.

In the right-alternative case we have $\operatorname{dim} \mathcal{R} \mathcal{A} l t(3)=9$, and the computations are similar. 


\section{Corollary 1.2.}

(i) A dual right-or left-alternative algebra over a field of characteristic different from 2 is nilpotent of degree 4.

(ii) A dual alternative algebra over a field of characteristic different from 2 and 3 is nilpotent of degree 6.

Proof: (i) We have, by subsequent application of associativity and (RA!),

$$
\begin{aligned}
(x y z) t & =-(x z y) t=-x(z y t)=x(z t y)=x(z t) y \\
& =-x y(z t) .
\end{aligned}
$$

(ii) Substituting $x=y=z$ in $\left(\mathrm{A}^{!}\right)$, we get $6 x^{3}=0$. The claim then follows from the results centered on the classical Dubnov-Ivanov-Nagata-Higman theorem about the nilpotency of associative nil algebras (see, for example, [Drensky 00, Section 8.3]).

These claims also could be proved with the help of Albert. $^{1}$

\section{DIMENSION SEQUENCE}

We are going to establish non-Koszulity using the well-known Ginzburg-Kapranov criterion [Ginzburg and Kapranov 94, Proposition 4.1.4(b)], which states that if a finitely generated binary quadratic operad $\mathcal{P}$ over a field of characteristic zero is Koszul, then

$$
g_{\mathcal{P}}\left(g_{\mathcal{P} !}(x)\right)=x,
$$

where

$$
g_{\mathcal{P}}(x)=\sum_{n=1}^{\infty}(-1)^{n} \frac{\operatorname{dim} \mathcal{P}(n)}{n !} x^{n}
$$

is the Poincaré series of the operad $\mathcal{P}$, and $\mathcal{P}^{!}$is the Koszul dual of $\mathcal{P}$. For this, we need to know the first few terms of the sequence $\operatorname{dim} \mathcal{P}(n)$ for the corresponding operads and/or their Koszul duals. This is achieved with the help of Albert.

Albert computes over a fixed prime field, and we are going to explain now how these computations imply results valid in characteristic zero.

Representing an operad $\mathcal{P}$ as the quotient of the free (= magmatic) operad $\mathcal{F}$ by the ideal of relations, and considering the corresponding arity- $n$ parts for a fixed $n$,

\footnotetext{
${ }^{1}$ The software Albert, version 4.0, is available online (http://
} www.cs.clemson.edu/ dpj/albertstuff/albert.html). we have

$$
\operatorname{dim} \mathcal{P}(n)+\operatorname{rk} M=\operatorname{dim} \mathcal{F}(n)=n ! C_{n-1},
$$

where $M$ is a matrix consisting of coefficients of all linear relations in $\mathcal{P}$ between all nonassociative multilinear monomials in $n$ variables.

Since the coefficients of the identities defining our operads are integers, $M$ is an integer matrix, and it is possible to consider its reduction $M_{p}$ modulo a given prime $p$.

It is clear that $\operatorname{rk} M \geq \operatorname{rk} M_{p}$. The question is how to ensure equality of these values. What follows is a variation on the standard theme in numerical linear algebra - how to replace rational or integer arithmetic by modular arithmetic.

Let us represent the matrix $M$ in the Smith normal form, i.e., as a product

$$
M=S \operatorname{diag}\left(d_{1}, \ldots, d_{r}, 0, \ldots, 0\right) T,
$$

where $S$ and $T$ are integer quadratic matrices with determinant equal to $\pm 1, r=\operatorname{rk} M$, and $d_{1}, \ldots, d_{r}$ are nonzero integers such that $d_{i+1}$ divides $d_{i}$. Reduction of this product modulo $p$ will produce the Smith normal form of $M_{p}$, i.e., $S_{p}, T_{p}$ are still matrices with determinant \pm 1 over $\mathbb{Z} / p \mathbb{Z}$, and the number of nonzero elements in the diagonal matrix

$$
\operatorname{diag}\left(d_{1}(\bmod p), \ldots, d_{r}(\bmod p), 0, \ldots, 0\right)
$$

is equal to $\operatorname{rk} M_{p}$.

If we pick primes $p_{1}, \ldots, p_{k}$ in such a way that

$$
p_{1} \cdots p_{k}>\left|d_{1} \cdots d_{r}\right|
$$

then

$$
d_{1} \cdots d_{r} \not \equiv 0\left(\bmod p_{1} \cdots p_{k}\right),
$$

and hence by the Chinese remainder theorem,

$$
d_{1} \cdots d_{r} \not \equiv 0\left(\bmod p_{i}\right),
$$

and hence $\operatorname{rk} M_{p_{i}}=\operatorname{rk} M$ for some $p_{i}$. Consequently, if $\operatorname{rk} M_{p_{i}}=r$ for all $i=1, \ldots, k$, then $\operatorname{rk} M=r$.

It remains to estimate $p_{1} \ldots p_{k}$ to ensure inequality $(2-2)$. The product $d_{1} \cdots d_{r}$ is equal, up to sign, to the determinant of a certain minor $Q$ of $M$ of size $r \times r$. Since the identities defining our operads have coefficients 1 or -1 , all nonzero elements of the matrix $M$ could be chosen to be equal to 1 or -1 , so the usual estimate in such situations is provided by the Hadamard inequality $|\operatorname{det}(Q)| \leq r^{r / 2}$ (see, for example, [Horn and Johnson 90, Section 7.8.2]). 
To summarize: if there are primes $p_{1}, \ldots, p_{k}$ such that Albert produces the same value

$$
\operatorname{dim} \mathcal{P}(n)=m
$$

modulo these primes, and

$$
p_{1} \cdots p_{k}>r^{r / 2}, \quad \text { where } r=n ! C_{n-1}-m,
$$

then $(2-3)$ holds over integers, and consequently over any field of characteristic zero.

Albert allows us to compute over a prime field $\mathbb{Z} / p \mathbb{Z}$ with $p \leq 251$. We have modified Albert to allow primes up to the largest possible value of the largest signed integer type, which is $2^{63}-1$ in standard modern computer architectures, both 32-bit and 64-bit. We also have modified it to facilitate batch processing. ${ }^{2}$

Since the time of Albert's computations turns out not to depend significantly on the value of the prime, to minimize the overall computation time, we are minimizing the number of Albert runs at the expense of larger primes, i.e., when choosing primes in the given range satisfying the condition (2-4), we are choosing primes as large as possible. This could be done with the help of PARI/GP. ${ }^{3}$

Using all this, we establish the following lemma:

Lemma 2.1. Over a field of characteristic zero, the first five terms of the sequence $\operatorname{dim} \mathcal{R} \mathcal{A l t}(n)$ are $1,2,9,60,530$.

Proof: Over any field, the first two values are obvious, and the third could be established by hand (in fact, we already did it in the proof of Proposition 1.1).

The are three primes less than $2^{63}$ satisfying the inequality $(2-4)$ for $r=4 ! C_{3}-60=60$ :

$$
2^{63}-259, \quad 2^{63}-165, \quad 2^{63}-25 .
$$

With these three primes, Albert produces $\operatorname{dim} \mathcal{R} \mathcal{A} l t(4)=$ 60 .

Similarly, the number of largest possible primes less than $2^{63}$ satisfying the inequality $(2-4)$ for $r=5$ ! $C_{4}-$ $530=1150$ is 93 , and Albert produces $\operatorname{dim} \mathcal{R} \mathcal{A} l t(5)=$ 530 for all these 93 primes.

We have also computed $\operatorname{dim} \mathcal{R} \mathcal{A} l t(6)=5820$ for a few random primes. ${ }^{4}$

\footnotetext{
${ }^{2}$ Albert version 4.0M (modified); available online (http:// justpasha.org/math/albert/).

${ }^{3} \mathrm{PARI} / \mathrm{GP}$ is available at http://pari.math.u-bordeaux.fr/.

${ }^{4}$ The corresponding sequence was submitted to the OnLine Encyclopedia of Integer Sequences (http://www. research.att.com/ njas/sequences/) as A161391.
}

Lemma 2.2. Over a field of characteristic zero, the first six terms of the sequence $\operatorname{dim} \mathcal{A l t}(n)$ are $1,2,7,32,175,1080 .^{5}$

Proof: We follow the same scheme as in the proof of Lemma 2.1. The corresponding number of primes is 5 for $n=4,127$ for $n=5$, and 3433 for $n=6$, and Albert produces the expected answers for all these primes.

The first five terms in Lemma 2.2 were already specified in [Loday 07], but the case $n=6$ is crucial. It requires the only time-consuming Albert computations among all computations mentioned in this note. We found that the optimal setting in this case was to add first the leftalternative identity, and then the right-alternative one, and use the static (as opposed to the sparse) matrix structure. The whole computation was finished in about a week running in parallel on a number of CPUs ranging from $2-\mathrm{GHz}$ single-core to $3.2-\mathrm{GHz}$ dual-core. The average execution time was less than one hour per prime.

\section{NON-KOSZULITY}

Theorem 3.1. The right-alternative, left-alternative, and alternative operads over a field of characteristic zero are not Koszul.

Proof: The statement for the right-alternative (leftalternative) case is known (and easy), but it will be instructive to look at it first and to compare it with the more difficult alternative case.

By Proposition 1.1 and Corollary 1.2(i), $\operatorname{dim} \mathcal{R} \mathcal{A} l t^{\prime}(3)=3$, and $\mathcal{R} \mathcal{A} l t^{!}(n)$ vanishes for $n \geq 4$, so the corresponding Poincaré series is

$$
g_{\mathcal{R} \mathcal{A} l t^{!}}(x)=-x+x^{2}-\frac{1}{2} x^{3} .
$$

On the other hand, by Lemma 2.1,

$$
g_{\mathcal{R} A l t}(x)=-x+x^{2}-\frac{3}{2} x^{3}+\frac{5}{2} x^{4}-\frac{53}{12} x^{5}+O\left(x^{6}\right),
$$

and

$$
g_{\mathcal{R} \mathcal{A} l t}\left(g_{\mathcal{R} \mathcal{A} l t^{\prime}}(x)\right)=x+\frac{1}{6} x^{5}+O\left(x^{6}\right),
$$

which contradicts Koszulity.

But in fact, we can establish the same without appealing to Lemma 2.1. Indeed, the beginning terms of

${ }^{5}$ The corresponding sequence was submitted to the On-Line Ency-
clopedia of Integer Sequences as A161392. 
the inverse to the polynomial $g_{\mathcal{R} \mathcal{A} l t^{\prime}}(x)$ are

$$
\begin{aligned}
- & x+x^{2}-\frac{3}{2} x^{3}+\frac{5}{2} x^{4}-\frac{17}{4} x^{5}+7 x^{6}-\frac{21}{2} x^{7}+\frac{99}{8} x^{8} \\
- & \frac{55}{16} x^{9}-\frac{715}{16} x^{10}+O\left(x^{11}\right) .
\end{aligned}
$$

The alternation of signs is violated at the tenth term; hence this series cannot be the Poincaré series of any operad, so by the Ginzburg-Kapranov criterion, $\mathcal{R} \mathcal{A} l t !$ is not Koszul, and hence $\mathcal{R} \mathcal{A} l t$ is not Koszul.

Moreover, the dimension sequence of $\mathcal{R} \mathcal{A} l t !$ coincides with the dimension sequence of the operad $\mathcal{P}$ relie $\bullet \mathcal{N} i l$ $(\mathcal{P}$ relie is the operad defined by a binary operation satisfying the pre-Lie (= right-symmetric) identity, $\mathcal{N} i l$ is the operad defined by a skew-symmetric binary operation with vanishing compositions, and $\bullet$ is Manin's black product), and the corresponding computation establishing its non-Koszulity was already performed in [Vallette 08, Section 4.5].

Now consider the alternative case. By Corollary 1.2(ii), $\mathcal{A} l t^{!}(n)$ vanishes for $n \geq 6$. Either computation with Albert or reference to [Lopatin 05, Propositions 1 and 2] provides dimensions of these spaces for small $n$, which allows us to write down the Poincaré series of the operad $\mathcal{A} l t^{!}$:

$$
g_{\mathcal{A} l t^{!}}(x)=-x+x^{2}-\frac{5}{6} x^{3}+\frac{1}{2} x^{4}-\frac{1}{8} x^{5} .
$$

On the other hand, by Lemma 2.2,

$$
\begin{aligned}
g_{\mathcal{A} l t}(x)= & -x+x^{2}-\frac{7}{6} x^{3}+\frac{4}{3} x^{4}-\frac{35}{24} x^{5} \\
& +\frac{3}{2} x^{6}+O\left(x^{7}\right)
\end{aligned}
$$

and

$$
g_{\mathcal{A l t}}\left(g_{\mathcal{A l t} !}(x)\right)=x-\frac{11}{72} x^{6}+O\left(x^{7}\right),
$$

which contradicts Koszulity.

Note that in the alternative case we really need to compute the dimension sequence of the alternative operad up to the sixth term (i.e., to utilize Lemma 2.2). A mere look at the inverse to the polynomial $g_{\mathcal{A l t}}(x)$ does not seem to work: we have checked with PARI/GP that the inverse has alternating signs up to degree 1000. On the other hand, as noted in [Goze and Remm 09, Section 4.2 , the beginning terms of the inverse to $g_{\mathcal{A l t}}(x)$ are

$$
-x+x^{2}-\frac{5}{6} x^{3}+\frac{1}{2} x^{4}-\frac{1}{8} x^{5}-\frac{11}{72} x^{6}+O\left(x^{7}\right),
$$

which provides an alternative proof of non-Koszulity of $\mathcal{A} l t$ without appealing to $g_{\mathcal{A l t}}(x)$.
Sometimes in the literature one sees expressed the viewpoint that non-Koszulity is a rather pathological property, and all "naturally" "occurring in real life" algebras should be algebras over a Koszul operad (see, for example, [Markl et al. 02, Remarks 3.98 and 3.131]). As we see, alternative algebras provide a "real-life" example violating this principle (another, albeit probably less "real life," contender is presented in [Dzhumadil'daev 09b]).

\section{POSITIVE CHARACTERISTIC}

The original Ginzburg-Kapranov operadic theory involves representation theory of the symmetric group peculiar to the characteristic-zero case. While extensions of the operadic theory to the case of positive characteristic exist, none of them, to our knowledge, includes an analogue of the Ginzburg-Kapranov criterion for Koszulity of a quadratic operad in terms of Poincaré series.

Therefore, while checking the validity of $(2-1)$ in positive characteristic does not make much sense, the question of computing the dimension sequence $\operatorname{dim} \mathcal{P}(n)$ for various operads $\mathcal{P}$ is still of interest. In this section we collect a few remarks and computational results concerning this question for the alternative and right-alternative operads and their Koszul duals.

For the Koszul dual operads, the corresponding dimension sequences terminate at low terms as indicated in the proof of Theorem 3.1, the same way for zero and positive characteristics, except for the case of the dual alternative operad over a field of characteristic 3 .

Conjecture 4.1. Over a field of characteristic 3, $\operatorname{dim} \mathcal{A} l t^{!}(n)=2^{n}-n$.

For $n \leq 8$ the claim could be proved with the aid of Albert. We will outline the main idea of a possible proof in the general case, whose full implementation appears to be long and somewhat cumbersome, and will drive us far away from the main question considered in this note. We came up with this idea by inspecting the corresponding entry A000325 in the On-Line Encyclopedia of Integer Sequences.

Sketch of a possible proof: For associative algebras, the identity $\left(\mathrm{A}^{!}\right)$is equivalent to the identity

$$
[[x, y], y]=0
$$

In other words, an associative algebra over a field of characteristic 3 is dual alternative if and only if its associated Lie algebra is 2-Engel. 
It is well known that 2-Engel Lie algebras are nilpotent of order 4 . Free associative algebras that are Lie-nilpotent of order 4 were studied in the recent paper [Etingof et al. 09].

It is possible to extend some of the results of that paper to the case of characteristic 3, and in particular, to construct a presentation of such algebras. From this, by adding more relations, one may construct a presentation of free dual alternative algebras, and using the composition (= diamond) lemma, obtain a description of a basis of such algebras in combinatorial terms. For elements of the basis containing each free generator in the first degree, these combinatorial terms are expressed as the so-called Grassmann permutations, i.e., $\mathcal{A} l t(n)$ has a basis consisting of associative monomials of the form $a_{i_{1}} \cdots a_{i_{n}}$ such that the permutation $\left(i_{1} \ldots i_{n}\right)$ has exactly one descent. The number of such permutations is $2^{n}-n$.

The case of characteristic 3 is also exceptional for the alternative operad: in this case, the first five terms of $\operatorname{dim} \mathcal{A} l t(n)$ are the same as in Lemma 2.2, while the sixth term is equal, surprisingly, to $1081 .^{6}$

Note also that the scheme of computations presented in Section 2 is insufficient to deduce the validity of (2-3) over all prime fields. Either by the standard ultraproduct argument, or observing, by the same argument as in Section 2 , that the equality $(2-3)$ in characteristic zero implies the same equality in characteristic $p$ for all $p>r^{r / 2}$, we may deduce that it is valid for all but a finite number of characteristics.

So in principle, we could establish the validity of $(2-3)$ in all characteristics by verifying it modulo all primes less than or equal to $r^{r / 2}$ and for one prime greater than $r^{r / 2}$. This is, however, computationally infeasible in almost all practical cases.

To be able to establish the equality $(2-3)$ in all characteristics, apparently other methods are needed. For example, one may try to use the capability of Albert to produce multiplication tables between elements of $\mathcal{P}(n)$ up to the given degree. It seems that the scheme, based on the Chinese remainder theorem and similar to those presented in Section 2, but utilizing the multiplication table instead of just dimensions of the corresponding spaces of multilinear monomials, could be used for that, provided that all coefficients in the computed multiplication tables are rational numbers with relatively small numer-

${ }^{6}$ The corresponding sequence was submitted to the On-Line Encyclopedia of Integer Sequences as A161393. ators and denominators modulo the respective primes. According to a few Albert computations we have performed for $\mathcal{A} l t(6)$, the latter seems to be the case for the alternative operad.

\section{QUESTIONS}

In addition to an example from [Vallette 08] already mentioned in Section 3, there are several other proofs in the literature of non-Koszulity of various operads using the Ginzburg-Kapranov criterion or its $n$-ary analogues: in [Getzler and Kapranov 95, footnote to Section 3.9(d)] for the so-called mock-Lie and mock commutative operads (which are Koszul dual to each other and are cyclic quadratic operads with one generator); in [Markl et al. 02, Remark 3.98] for associative anticommutative algebras (and, dually, for "commutative Lie algebras"); in [Goze and Remm 04, Proposition 2.3] for certain Lie-admissible operads dubbed $G_{4}-A s s$ and $G_{5}-A s s$; in [Goze and Remm 09, Sections 3.4, 3.6] for certain third-power associative operads dubbed $G_{i}-p^{3} A s s$; in [Dzhumadil'daev 09a, Theorem 10.1] for a certain skewsymmetric operad dubbed left-Alia; in [Dzhumadil'daev 09b] for the Novikov operad; and in [Markl and Remm 09, Example 16 and Proposition 17] for certain operads with $n$-ary operation dubbed $t A s s_{d}^{n}$.

In each of these cases, it was enough to check Poincaré series up to a term of relatively low degree. It is of interest whether there exists a bound on the degree of Poincaré series such that the validity of the identity $(2-1)$ for a binary quadratic operad $\mathcal{P}$ up to this degree guarantees its validity in all degrees.

It would also be of interest to give a concrete example of a binary quadratic operad that is not Koszul but for which the equality $(2-1)$ holds (such examples exist for associative quadratic algebras - see [Polishchuk and Positselski 05, Section 3.5] and references therein).

Is it true that all terms of the inverse of the polynomial (3-1) have alternating signs? If yes, what combinatorial interpretation might this have? (This question was posed by Vladimir Dotsenko.)

A similar question about an innocent-looking polynomial of degree 15 and with only three nonzero terms was asked in [Markl and Remm 09]. Somewhat surprisingly, such questions seem to be difficult.

Note also that it remains a challenging problem to compute the Poincaré series of $\mathcal{A} l t$.

And finally, we are taking the opportunity to advertise some new classes of algebras. In [Dzhumadil'daev 09a, Theorem 5.1], all possible skew-symmetric identities of 
degree 3 are classified. This classification has a symmetric analogue: every symmetric identity of degree 3 can be reduced to one of the following identities:

$$
\begin{array}{r}
{[\{x, y\}, z]+[\{y, z\}, x]+[\{z, x\}, y]=0,} \\
\{\{x, y\}, z\}+\{\{y, z\}, x\}+\{\{z, x\}, y\}=0, \\
\{x, y\} z+\{y, z\} x+\{z, x\} y=0,
\end{array}
$$

where $[x, y]=x y-y x$ and $\{x, y\}=x y+y x$. Any rightor left-alternative algebra satisfies the first of these identities, and the second identity is exactly $\left(\mathrm{A}^{!}\right)$(with appropriately inserted left-normed brackets, since associativity is no longer assumed). It appears to be of interest to study algebras satisfying these identities, in particular to describe free and simple algebras in these classes and to look at the corresponding operads.

\section{ACKNOWLEDGMENTS}

Thanks are due to Vladimir Dotsenko and Leonid Positselski for explanations concerning Koszulity and the Ginzburg-Kapranov criterion, to Alexander Feldman for advice concerning computing issues and comments on the preliminary version of the manuscript, and to the anonymous referee for a very careful reading of the manuscript and useful comments as well.

\section{REFERENCES}

[Baez 02] J. Baez. "The Octonions." Bull. Amer. Math. Soc. 39 (2002), 145-205; Errata: 42 (2005), 213.

[Drensky 00] V. Drensky. Free Algebras and PI-Algebras. New York: Springer, 2000.

[Dzhumadil'daev 09a] A. S. Dzhumadil'daev. "Algebras with Skew-Symmetric Identity of Degree 3." J. Math. Sci. 161 (2009), 11-30.

[Dzhumadil'daev 09b] A. S. Dzhumadil'daev. "Codimension Growth and Non-Koszulity of Novikov Operad." To appear in Comm. Algebra. Available online (arXiv:0902.3771v1), 2009.
[Etingof et al. 09] P. Etingof, J. Kim, and X. Ma. "On Universal Lie Nilpotent Associative Algebras." J. Algebra 321 (2009), 697-703.

[Getzler and Kapranov 95] E. Getzler and M. Kapranov. "Cyclic Operads and Cyclic Homology." In Geometry, Topology and Physics for Raoul Bott, edited by S.-T. Yau, Conf. Proc. Lect. Notes Geom. Topol. 4, pp. 167-201. Cambridge, MA: International Press, 1995.

[Ginzburg and Kapranov 94] V. Ginzburg and M. Kapranov. "Koszul Duality for Operads." Duke Math. J. 76 (1994), 203-272; Erratum: 80 (1995), 293.

[Goze and Remm 04] M. Goze and E. Remm. "Lie-Admissible Algebras and Operads." J. Algebra 273 (2004), 129-152.

[Goze and Remm 09] M. Goze and E. Remm. "A Class of Nonassociative Algebras Including Flexible and Alternative Algebras, Operads and Deformations." arXiv:0910.0700v1, 2009.

[Horn and Johnson 90] R. Horn and C. Johnson. Matrix Analysis. Cambridge, UK: Cambridge Univ. Press, 1990.

[Loday 07] J.-L. Loday, Encyclopedia of Types of Algebras. Available online ( http://www-irma.u-strasbg.fr/ loday/jllpub.html), 2007.

[Lopatin 05] A. A. Lopatin. "Relatively Free Algebras with the Identity $x^{3}=0 . "$ Comm. Algebra 33 (2005), 3583-3605.

[Markl and Remm 09] M. Markl and E. Remm. "(Non-) Koszulity of Operads for $n$-ary Algebras, Cohomology and Deformations." arXiv:0907.1505v1, 2009.

[Markl et al. 02] M. Markl, S. Shnider, and J. Stasheff. Operads in Algebra, Topology and Physics. Providence: AMS, 2002.

[Polishchuk and Positselski 05] A. Polishchuk and L. Positselski. Quadratic Algebras. Providence: AMS, 2005.

[Vallette 08] B. Vallette, "Manin Products, Koszul Duality, Loday Algebras and Deligne Conjecture." J. Reine Angew. Math. 620 (2008), 105-164.

Askar Dzhumadil'daev, Kazakh-British Technical University, Tole bi 59, Almaty 050000, Kazakhstan (dzhuma@hotmail.com)

Pasha Zusmanovich, Department of Mathematics, Tallinn University of Technology, Ehitajate tee 5, Tallinn 19086, Estonia (pasha.zusmanovich@ttu.edu)

Received July 4, 2009; accepted March 3, 2010. 